\title{
On Channel Correlation Based Scheduling and Signalling for MIMO-OFDMA Downlink
}

\author{
Andreas Ibing, Holger Boche \\ Chair of Theoretical Information Technology \\ Technische Universität München \\ Arcisstrasse 21, 80333 München, Germany
}

\author{
Philipp Otto \\ Heinrich-Hertz-Chair for Mobile Communcations \\ Technische Universität Berlin \\ Einsteinufer 25, 10587 Berlin, Germany
}

\begin{abstract}
For joint MIMO-OFDMA link adaptation and scheduling, channel quality feedback needs to be quantized, sparse and irregular. For flexible scheduling, prediction of the quantized channel at the base-station is needed. We propose measurement of 3D channel correlation parameters by the terminals and signalling back to the base station, which enables prediction of the quantized channel by multi-dimensional Wiener filtering. The scheme is shown to improve adaptive choice of transmission parameters and to avoid mis-adaptation due to control lag. Different schemes of correlation feedback signalling are discussed in terms of (time-variant) expected throughput.

Index Terms-MIMO-OFDMA, Feedback, Prediction
\end{abstract}

\section{INTRODUCTION}

Time variance of a radio channel is divided into largescale fading due to pathloss and shadowing and smallscale fading due to multipath propagation (changes over distance on the order of wavelength). Large-scale fading is traditionally countered by adaptation of transmit power. Adaptation of transmit parameters on a radio link has since been extended to also account for small-scale fading, e.g. in the form of per-subcarrier modulation in adaptive OFDM [1]. Link adaptation as any control loop needs to consider adaptation delay. To cope with delay, channel prediction for adaptive OFDM based on received symbols is described e.g. in [2]-[4] using Wiener filtering or Kalman filtering respectively. Link adaptation either follows a channel reciprocity assumption for time division duplex (TDD) links, or requires a feedback channel to report the fading behaviour (closed-loop adaptation) and possibly also interference. To reduce feedback bandwidth, channel values can be quantized to yield the parameters of adaptation [2]. Modern MIMOOFDMA transmission further extends the trend towards finer-grained adaptation in two directions. In OFDMA, link adaptation can be combined with multi-user scheduling to exploit multi-user diversity in addition to time and frequency diversity [5]. In MIMO transmission, MIMO parameters can be adapted depending on channel correlation between different antennas [6]. Joint multi-user MIMO scheduling and link adaptation is described e.g. in [7].

Feedback for the complete channel grows linearly with the number of users, while the corresponding capacity grows

This work was accomplished while the authors were at Heinrich-HertzChair for Mobile Communications, TU Berlin, Germany. only double logarithmically [7] - so further reduction of feedback information is necessary for joint scheduling and link adaptation. For this reduction, usage of SNR thresholds has been proposed to avoid transmitting feedback for 'bad' channel ressources [8], [9]. These thresholds can be adaptive, relative (quality ordering) and individual per user. [7] evaluates feedback thresholds to achieve a sum feedback rate limitation. A further overview of limited feedback schemes is given in [10]. Prediction in the base station based on quantized feedback is discussed in [11]. It uses linear predictive coding, where the filter parameters are determined by the base station.

A recent commercial adaptation and signalling scheme for FDD MIMO-OFDMA is given by LTE [12]. Downlink channel measurement uses the pilot symbols for coherent demodulation, feedback regarding the downlink channel is transmitted over an uplink control channel in the form of wideband or subband feedback (subband selection either by base station or by terminal). The feedback consists of channel quality indication (modulation and code rate), rank index (number of MIMO streams) and precoding matrix indication (for MIMO stream selection), depending on transmission mode. Uplink measurement uses a sounding signal, where the bandwidth and antennas are commanded by the base station. Feedback or sounding transmission are thus a description of (part of) the actual space-frequency quantized channel. Time instances for feedback and sounding are commanded by the base station, either as periodic or aperiodic. Signalling overhead is further reduced by the possibility for persistent scheduling.

So on the one hand, flexible scheduling and link adaptation need channel information for all ressources. On the other, reduced feedback for MIMO-OFDMA needs to be quantized, sparse, and irregular (also due to changing traffic requirements). Collisions of subband and stream selection can occur. To resolve these contradicting requirements as well as the adaptation delay problem, prediction of the quantized channel is necessary. For reduced feedback overhead, this prediction should be performed at the base station. To enable this prediction, we propose to also feedback channel correlation information (which changes slower than the channel itself). Prediction (including error prediction) based on non-uniform sampling (feedback) positions can be 


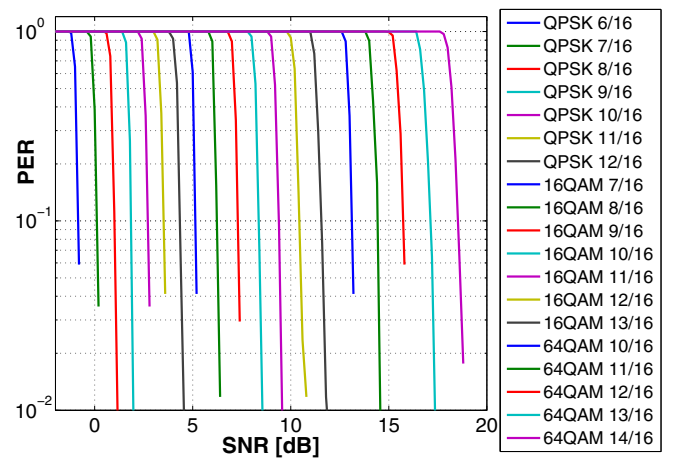

Fig. 1. Packet error rates for different transmission modes (modulation and code rate), chosen to have $1 d B$ distance.

performed by Wiener filtering and is an approach to avoid mis-adaptation in wireless systems covering a wide range of mobility and deployment (location) scenarios.

\section{Benefit of Channel CoRrelation KNOWledge}

We denote the time-variant channel transfer function as $h(i, j, p)$, with $i$ OFDM symbol index, $j$ subcarrier index and $p$ pair of transmit / receive antennas. For link adaptation and scheduling we are rather interested in the quantized channel according to granularity of adaptation / scheduling: a physical ressource block (PRB) consists of $N_{s u b}$ subcarriers over $N_{t}$ OFDM symbols. The average squared channel gain of a PRB is:

$$
\bar{g}(t, f, p)=\frac{1}{N_{s u b} N_{t}} \sum_{i \in \mathrm{PRB}} \sum_{t \in \mathrm{PRB} f}|h(i, j, p)|^{2}
$$

with $t$ PRB time index and $f$ PRB frequency index. The correlation is

$$
\begin{aligned}
R_{\bar{g}}\left(\Delta t, \Delta f, p_{1}, p_{2}\right)= & \frac{E\left[\left(\bar{g}\left(t, f, p_{1}\right)-\mu_{g}\right)\right.}{\sigma_{g}} . \\
& \cdot \frac{\left.\left(\bar{g}\left(t+\Delta t, f+\Delta f, p_{2}\right)-\mu_{g}\right)\right]}{\sigma_{g}}
\end{aligned}
$$

where $\bar{g}$ is assumed as stationary random process with mean $\mu_{g}$ and variance $\sigma_{g}$. As in [13] we assume that the correlation is separable into three factors:

$$
R_{\bar{g}}\left(\Delta t, \Delta f, p_{1}, p_{2}\right)=r_{t}(\Delta t) r_{f}(\Delta f) r_{s}\left(p_{1}, p_{2}\right)
$$

Autocorrelation is the inverse Fourier transform of power spectral density.

$$
r_{t}(\Delta t) r_{f}(\Delta f)=\mathcal{F}^{-1}\left(S_{\text {del }}(\tau) S_{D o p}(\nu)\right)
$$

with Doppler power spectrum spectrum $S_{D o p}(\nu)$ and delay power spectrum $S_{d e l}(\tau)$. Common Doppler spectrum assumptions for the channel $h$ include Gaussian shape, uniform spectrum or Jakes spectrum [14]. Precondition for correlation signalling is of course estimation of the correlation by the terminal - but high-performance channel estimation by adaptive filtering requires the receiver to estimate channel correlation anyway [15]. To predict the

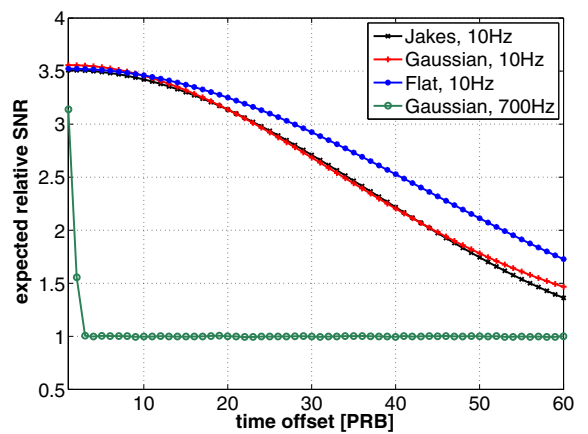

Fig. 2. 20MHz Urban macro OFDM channel's frequency selection gain in dependence on signalling delay, for different Doppler spectrum shapes and maximum Doppler frequencies.

expected value $\mu_{p}$ of $\bar{g}$ on a position $(t, f, p 1, p 2)$ from a vector $\mathbf{f}$ of (possibly non-uniform) feedback samples, we use the Wiener filter [16]:

$$
\hat{\mu_{p}}=\left(\mathbf{R}_{\mathcal{A}}^{-1} \mathbf{r}_{\mathcal{A}}\right)^{T}\left(\mathbf{f}-\mu_{g} \mathbf{1}\right)+\mu_{g}
$$

where $\mathbf{R}_{\mathcal{A}}$ is the correlation matrix of the feedback samples (within filter area $\mathcal{A}$ ), $\mathbf{r}_{\mathcal{A}}$ is the correlation vector of these sample positions and the position to predict, and $\mathbf{1}$ is a vector of adequate size containing ones. The process mean $\mu_{g}$ is subtracted from the feedback and added later to the prediction. The corresponding prediction error has the variance [17]:

$$
\hat{\sigma_{p}^{2}}=\sigma_{g}^{2}\left(1-\mathbf{r}_{\mathcal{A}}^{T} \mathbf{R}_{\mathcal{A}}^{-1} \mathbf{r}_{\mathcal{A}}\right)
$$

The prediction is quite similar to multidimensional Wiener interpolation filtering for pilot symbol assisted channel estimation as in [17] (where in this case we have nonzero process mean and non-uniform sampling). For single feedback, $\mathbf{R}_{\mathcal{A}}=1$ and $\hat{\mu_{p}}$ decreases with the time-direction autocorrelation $\mathbf{r}_{t}(\Delta t)$. With growing time lag between feedback positions and PRB to predict, the mean prediction converges to the process mean, and the error prediction to the process variance [18]:

$$
\lim _{\Delta t \rightarrow \infty} \hat{\mu}_{p}=\mu_{g}, \quad \lim _{\Delta t \rightarrow \infty} \hat{\sigma}_{p}=\sigma_{g}
$$

1) Time-direction autocorrelation: autocorrelation of $\bar{g}$ as described by power Doppler spectrum can be approximated using a parametric model with few parameters, in the first approximation (one parameter) e.g. terminal speed, maximum Doppler shift or channel coherence time (correlation dropped to $50 \%$ ). We illustrate with three common oneparametric models. Using the Jakes model, Doppler spectrum and correlation are [14]:

$S(f)=\frac{1}{\pi f_{d} \sqrt{1-\left(f / f_{d}\right)^{2}}},|f| \leq f_{d} ; r_{t}(\Delta t)=J_{0}\left(2 \pi f_{d} \Delta t\right)$

with $f_{d}$ for the maximum Doppler shift.

The Gaussian spectrum assumption is:

$$
S(f)=\frac{1}{\sqrt{2 \pi \sigma^{2}}} e^{-\frac{f^{2}}{2 \sigma^{2}}} ; \quad r_{t}(\Delta t)=e^{-\pi \sigma \sqrt{2} \cdot(\Delta t)^{2}}
$$




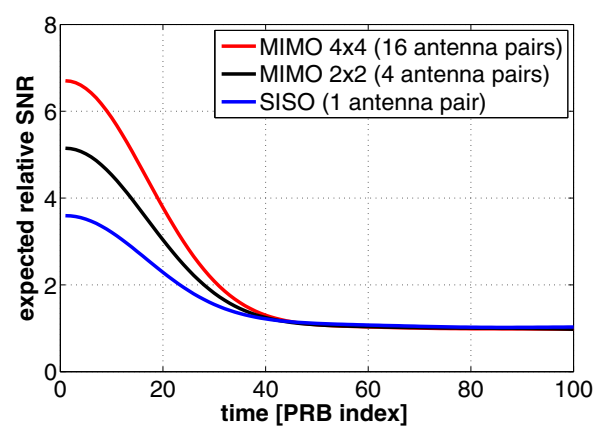

Fig. 3. MIMO-OFDMA space-frequency selection gain over delay for uncorrelated channels $(20 \mathrm{~Hz}$ max. Doppler, Gauss spectrum, $20 \mathrm{MHz}$ urban macro).

where $f_{d}$ is defined as $f_{d}=\sigma \sqrt{2}$, and $\sigma$ is the standard deviation.

For the uniform model we have:

$$
S(f)=\frac{1}{2 f_{d}},|f| \leq f_{d} ; \quad r_{t}(\Delta t)=\operatorname{sinc}\left(2 f_{d} \Delta t\right)
$$

For single feedback and time-direction prediction, the expected adaptation gain in dependence on feedback delay is illustrated in Fig. 2 for different spectra of $h$. Simulation uses the urban macro channel model [19], a PRB spans $0.5 \mathrm{~ms}$ times $180 \mathrm{kHz}$ (as in LTE). The expected adaptation gain by selecting the best $\mathrm{PRB}$ in frequency direction (compared to channel average) is 3.5, for zero delay. With increasing delay (round-trip and protocol delay) the figure shows the descent to channel average. The three example models (Jakes, Gaussian, uniform) show little difference for the same max. Doppler shift (in the figure $10 \mathrm{~Hz}$, corresponding to low mobility, $5 \mathrm{~km} / \mathrm{h}$ at $2 \mathrm{GHz}$ ). The figure also shows the expected adaptation gain over delay for $700 \mathrm{~Hz}$ (medium/high mobility, $60 \mathrm{~km} / \mathrm{h}$ at $2 \mathrm{GHz}$ ), which reduces the coherence time reduced from around $40 \mathrm{PRBs}$ to less then 1 (anti-proportional to $f_{D}$ ). Fig. 3 illustrates the expected adaptation gain over delay if the best PRB (urban macro channel) is selected not only in frequency direction, but also over different (uncorrelated) MIMO streams (Gaussian spectrum, $f_{D}=20 \mathrm{~Hz}$ ). While the expected adaptation gain increases with the number of uncorrelated antenna pairs, the behaviour over time is not influenced - the larger relative decrease poses even a higher risk for misadaptation. In the following we determine packet error rate (PER) and throughput in dependence on signalling (adaptation) lag. Fig. 1 illustrates PER for different transmission modes (of one spatial stream; modulation and code rate are varied). With current rate matching methods (e.g. [20]), the code rate can be chosen with very fine granularity. With strong coding (LTE turbo code in the example), the PER show an 'onoff' behaviour in dependence on SNR: at a threshold SNR the PER drops very fast. The transmission modes in Fig. 1 are chosen to have a spacing of approximately $1 \mathrm{~dB}$ SNR. In addition to the expected mean adaptation gain as in Fig. 2, Fig. 5 also shows the expected variance in dependence

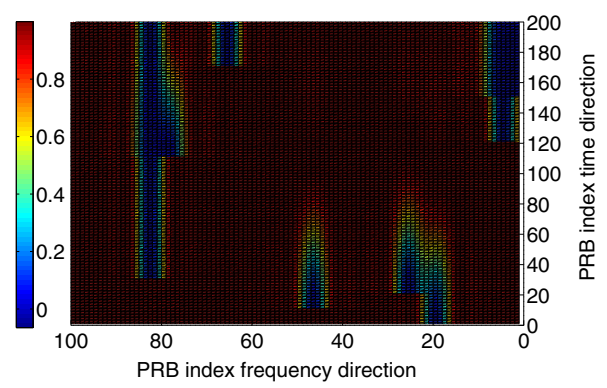

Fig. 4. Prediction error variance for periodic feedback about best PRB in frequency direction (every $10 \mathrm{PRB}$ ) and example channel realisation.

on delay, i.e. the expected prediction of mean value and prediction error according to Eq. (5) and Eq. (6) (for urban macro channel parameters and a Doppler spectrum assumption). The expected channel gain for a certain delay $\Delta t$ is described by a Gaussian distribution $\mathcal{N}(\mu(\Delta t), \sigma(\Delta t))$. The figure also contains SNR thresholds $T_{m}$ for different transmission modes $m$ ( $1 \mathrm{~dB}$ distances). With this, the PER in dependence on delay can be determined as:

$$
\begin{aligned}
\operatorname{PER}\left(T_{m}, \Delta t\right) & =\int_{-\infty}^{T_{m}} \mathcal{N}(\mu(\Delta t), \sigma(\Delta t)) \\
& =\Phi\left(\frac{T_{m}-\mu(\Delta t)}{\sigma(\Delta t)}\right)
\end{aligned}
$$

with the tail distribution $\Phi$. The resulting PER for different transmission modes is depicted in Fig. 6 in dependence on delay. A transmission mode $m$ maps $B_{m}$ bits into a PRB, so the expected relative throughput compared to mode $m_{1}$ is:

$$
\operatorname{TP}(m, \Delta t)=\frac{B_{m} \cdot \operatorname{PER}(T, \Delta t)}{B_{m_{1}}}
$$

Resulting throughput for different modes and delays is illustrated in Fig. 7. We propose that terminals feed back current parameters of the Doppler spectrum model to the base station, so that the base station can choose the transmission mode to optimize expected throughput, considering the control lag. To illustrate the benefit of time-direction correlation knowledge, we compare link adaptation for periodic channel quality feedback with and without correlation knowledge. Following a (short-term) static channel assumption (channel correlation not known), the expected average throughput in dependence on delay $t_{l a g}$ and feedback period $t_{p e r}$ is:

$$
\overline{\mathrm{TP}}_{\text {static }}\left(m, t_{\text {lag }}, t_{\text {per }}\right)=\frac{1}{t_{\text {per }}} \sum_{\Delta t=t_{\text {lag }}}^{t_{\text {lag }}+t_{\text {per }}} \mathrm{TP}(m, \Delta t)
$$

With known correlation and the same feedback scheme, the average expected throughput becomes:

$$
\overline{\operatorname{TP}} \overline{c o r r}_{\left.t_{\text {lag }}, t_{p e r}\right)}=\frac{1}{t_{\text {per }}} \sum_{\Delta t=t_{\text {lag }}}^{t_{\text {lag }}+t_{\text {per }}} \max _{m} \operatorname{TP}(m, \Delta t)
$$




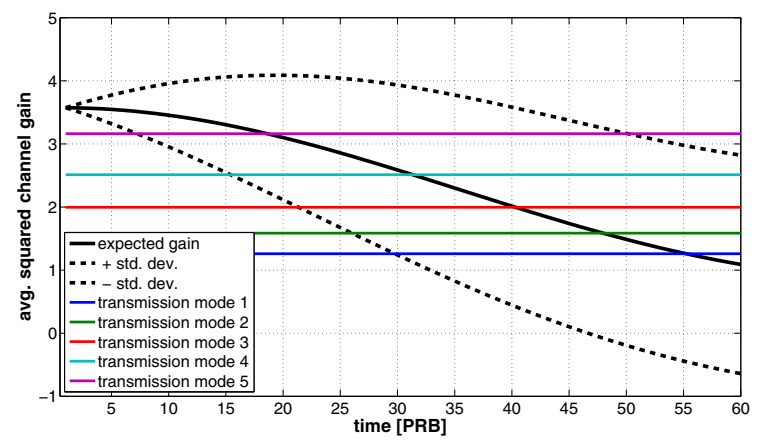

Fig. 5. Mean and standard deviation of adaptation gain over delay, also transmission mode SNR thresholds ( $1 d B$ spacing).

The expected best transmission mode can be chosen in dependence on delay, which corresponds to the envelope in Fig. 7.

2) Correlation in frequency direction and over antennas: There are several reasons for the benefit of knowledge about the channel's 3D correlation. First, more correlation between feedback samples improves prediction accuracy (compare Eq. (6)). Second, with a growing number of users, the probability of collisions of user-selected subband feedback increases - which necessitates prediction for positions also on other frequencies and/or streams. In addition, spatial (antenna) correlation can be used to select the number of MIMO streams and/or MIMO mode. For 3D Wiener prediction filtering, the equations (3), (5), (6) remain unchanged - only the filter area increases in dimensionality. Fig. 4 illustrates the prediction error for 2D prediction (time-frequency, OFDM channel) for periodic feedback with $t_{\text {per }}=10$. Other parameters for this illustration are Gaussian Doppler spectrum with $f_{D}=10 \mathrm{~Hz}$, uniform delay spectrum with $\tau_{\max }=0.5 \mu \mathrm{s}$, and zero signalling delay. For feedback positions the prediction error is zero, and with increasing distance from feedback positions, the prediction error increases towards process variance.

\section{SignALling AND TRACKING}

We assume now that the terminal performs measurement of the short-time 3D correlation of $\bar{g}$ over a window in time direction (on the order of coherence time), frequency and angular direction (or equivalently between antenna pairs) e.g. according to [15]. To include feedback signalling into throughput optimization, the throughput has to be reduced by signalling overhead. If asymmetric power and energy constraints are neglected in an FDD system, then it is reasonable to reduce downlink throughput by the corresponding feedback overhead on the uplink (since the frequency splitup in downlink/uplink could be adjusted). Alternatively, different weights can be given for uplink versus downlink bits.

3) Feedback reporting parameters: While the cost of feedback signalling is clear, the benefit is the expected throughput increase with the additional feedback (expected

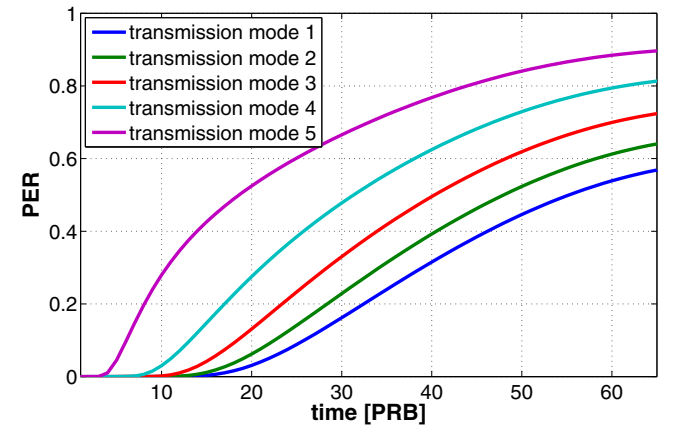

Fig. 6. Packet error rates over delay for the transmission modes from Fig.

throughput conditioned on feedback positions). For single feedback (request and transmission) this can be determined by Eq. (5) and (12), for periodic feedback directly from Eq. (14). The expected adaptation gain depends of course on the multi-variate probability density function of the channel (compare Fig. 2 and Fig. 3). Assuming a constant bit rate source and periodic feedback, the feedback period could be optimized by:

$$
t_{\text {per }}^{(\text {opt })}=\underset{t_{\text {per }}}{\operatorname{argmax}}\left(\overline{\mathrm{TP}}_{\text {corr }}\left(t_{\text {per }}\right)-\mathrm{OV}\left(t_{\text {per }}\right)\right)
$$

where the feedback overhead $\mathrm{OV}\left(t_{\text {per }}\right)$ is a hyperbola. A more detailed model may include feedback collision probability and expected throughput for alternative allocations.

4) Time-variant channel autocorrelation: Channel correlation changes slower than the channel itself (by definition and window-based estimation), so separate signalling for channel correlation and channel quality feedback reporting is adequate. While time-direction correlation $r_{t}$ is velocity dependent, frequency- and space-direction correlation are location dependent and could possibly be stored in the base station to avoid signalling. The maximum Doppler shift in dependence on terminal velocity is $f_{d}=f_{c} \cdot \frac{v}{c}$, with $f_{c}$ carrier frequency, $v$ velocity, and $c$ speed of light. Time-direction correlation change due to acceleration $\frac{d v}{d t}$ (assuming same spectrum shape) with acceleration values achieved by cars means a slow correlation change which can be tracked by low-rate correlation change signalling. A different cause of changing correlation can be variation of the spectrum shape due to alterations of reflectors and scatterers in the channel (e.g. passing cars, trains). Different ways of correlation change signalling are discussed in the following and illustratied in Fig. 8. For the example scenario, the urban macro channel model and round-trip delay of 10 PRB (5ms) is assumed, further a Gaussian Doppler spectrum and (non-continuous) change of $f_{D}$ according to a step function from $10 \mathrm{~Hz}$ to $200 \mathrm{~Hz}$. Other parameters are periodic channel quality feedback (3 PRB period) and correlation measurement delay (3 PRB for $200 \mathrm{~Hz}$ ). The blue curve in Fig. 8 shows the expected throughput over time for no correlation signalling and (short-time) static channel assumption. The red curve shows the expected 


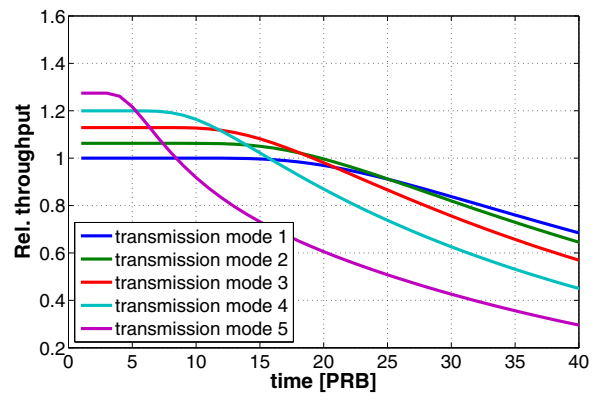

Fig. 7. Throughput over delay for the transmission modes from Fig. 5.

throughput for periodic correlation reporting with 10 PRB period. As already seen in Sec. II, correlation signalling improves the steady-state throughput, which for higher $f_{D}$ is smaller. With periodic correlation reporting, the transmission is adapted to the changed correlation after measurement delay, average reporting delay (average offset of reporting time to correlation change time) and round-trip delay.

5) Terminal-decided aperiodic update of correlation information: To minimize both correlation reporting delay and overhead, it is desirable to update the reported correlation only when a (significant) change has occurred: this means terminal-decided correlation feedback reporting. Terminal-decided aperiodic feedback transmission requires that an allocated uplink ressource is available (data or control channel). Implementation can be transmission of correlation information instead of scheduled data, or possibly piggy-back to channel quality reporting. Terminaldecided feedback offers an overhead reduction compared to high feedback periodicity, and packet error rate reduction compared to updating with low periodicity. The black curve in Fig. 8 illustrates expected throughput for terminal-decided correlation feedback, which compared to periodic correlation feedback avoids the average reporting delay.

\section{Discussion}

Jointly considering downlink and uplink, there is an 'information discrepency' between the base station and a terminal: while each terminal can continuously track the downlink channel, the base station can estimate an uplink channel only on resources where the terminal transmits (data, control or sounding). So the proposed method to obtain correlation information in this paper was measurement be the terminal and feedback signalling. The channel correlation parameters of downlink and uplink are themselves correlated, because changes in channel autocorrelation are caused by the same physical reasons (acceleration, location). This means both that a terminal can also continuously estimate correlation of the uplink channel, and that the base station could infer downlink correlation parameters from uplink sounding (if the sounding signal is transmitted with low period) which could be an alternative method to gain correlation information even without signalling.

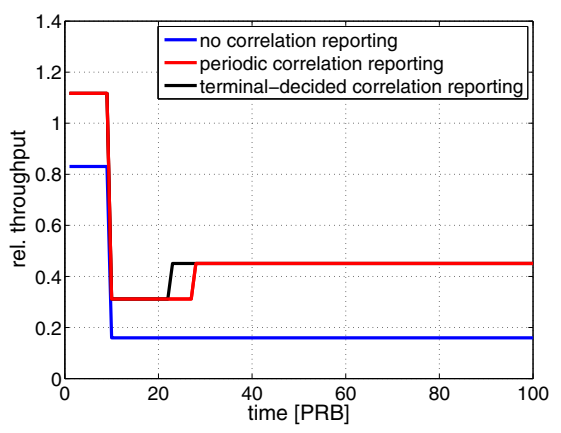

Fig. 8. Expected throughput over time for step function correlation change (after 10 PRB) and different correlation signalling schemes.

\section{REFERENCES}

[1] A. Czylwik, "Adaptive OFDM for wideband radio channels," in IEEE Globecom, 1996, pp. 713-718.

[2] T. Keller and L. Hanzo, "Adaptive multicarrier modulation: A convenient framework for time-frequency processing in wireless communications," Proceeding of the IEEE, vol. 88, no. 5, May 2000.

[3] M. Münster and L. Hanzo, "MMSE channel prediction assisted symbol-by-symbol adaptive OFDM," in IEEE ICC, 2002, pp. 416420.

[4] M. Sternad and D. Aronsson, "Channel estimation and prediction for adaptive OFDM downlink," in IEEE VTC fall, 2003, pp. 1283-1287.

[5] I. Wong and B. Evans, "Optimal downlink OFDMA resource allocation with linear complexity to maximize ergodic rates," IEEE Trans. Wireless Communications, vol. 7, no. 2, Feb. 2008.

[6] A. Forenza, M. McKay, A. Pandharipande, R. Heath, and I. Collings, "Adaptive MIMO transmission for exploiting the capacity of spatially correlated channels," IEEE Trans. Vehicular Technology, vol. 56, no. 2, March 2007.

[7] K. Huang, R. Heath, and J. Andrews, "Space division multiple access with a sum feedback rate constraint," IEEE Trans. Signal Processing, vol. 55, no. 7, pp. 3879-3891, July 2007.

[8] T. Bonald, "A score-based opportunistic scheduler for fading radio channels," in European Wireless Conference, 2004.

[9] V. Hassel, M. Alouini, D. Gesbert, and G. Oien, "Exploiting multiuser diversity using multiple feedback thresholds," in Proc. IEEE Veh. Technol. Conf., 2005.

[10] D. Love, R. Heath, V. Lau, D. Gesbert, B. Rao, and M. Andrews, "An overview of limited feedback in wireless communication systems," IEEE JSAC, vol. 26, no. 8, Oct. 2008.

[11] B. Mielczarek and W. Krzymien, "Adaptive CSI prediction in linear multi-user MIMO systems," in IEEE VTC fall, 2009.

[12] 3GPP TSG RAN: TS36.300v.10.0.0 E-UTRA and E-UTRAN; Overall Description; Stage 2, 3GPP, June 2010.

[13] S. Kim and Y. Lee, "3-dimensional MMSE channel estimation in multi-antenna OFDM systems," in IEEE Int. Conf. Digital Telecommunications, 2008.

[14] M. Patzold, Mobile Fading Channels, 1st ed. Wiley, 2002

[15] K. Jeong and J. Lee, "Low complexity channel tracking for adaptive MMSE channel estimation in OFDM," in IEEE CISS, 2007.

[16] N. Wiener, Extrapolation, Interpolation, and Smoothing of Stationary Time Series. Wiley, 1949.

[17] P. Hoeher, S. Kaiser, and P. Robertson, "Two-dimensional pilotsymbol-aided channel estimation by Wiener filtering," in IEEE ICASSP, 1997.

[18] P. Brockwell and R. Davis, Time Series: Theory and Methods. Springer, 1987.

[19] TSG RAN: TR25.814v7.1.0 Physical layer aspects for Evolved Universal Terrestrial Radio Access, 3GPP, Sept 2006.

[20] J. Cheng, A. Nimbalker, Y. Blankenship, B. Classon, and T. Blankenship, "Analysis of circular buffer rate matching for LTE turbo code," in IEEE VTC, 2008fall. 